\title{
Tunable composite nanoparticle for plasmonics
}

\author{
Gaëtan Lévêque and Olivier J.F. Martin \\ Nanophotonics and Metrology Laboratory, École Polytechique Fédéral de Lausanne, STI-NAM, ELG Station 11, \\ CH-1015 Lausanne, Switzerland
}

Received April 5, 2006; revised July 5, 2006; accepted July 6, 2006; posted July 10, 2006 (Doc. ID 69673); published August 25, 2006

We present a numerical study of the tunability properties of a plasmonic subwavelength particle deposited on a metallic slab. The particle is composed of a metallic part, supporting a localized plasmon mode, separated from the slab by a dielectric spacer. It is shown that the position of the resonance wavelength can be modified over a large spectral range by changing either the spacer thickness by a few tens of nanometers or its susceptibility within the range of usual dielectrics. A linear relation is observed between the resonance wavelength and the spacer permittivity. (C) 2006 Optical Society of America

OCIS codes: $130.3120,220.4830,240.6680,260.3910$

Plasmon resonances are electromagnetic modes associated with the excitation of collective oscillations of the electronic charge density in metals. They combine both high-field intensities and frequency selectivity and therefore could be used for new photonic devices integrated on very small surfaces. Two types of plasmon resonance exist: surface plasmon polariton (SPP) waves propagating along a metaldielectric interface and localized surface plasmon (LSP) modes, confined to subwavelength metallic objects. ${ }^{1,2}$ Individual particles can be assembled into small or complex arrangements such as chains or gratings where complex plasmonic functions can be achieved. Furthermore, the strong optical fields generated in these systems can in turn be used to enhance specific optochemical effects, such as surface enhanced Raman scattering, to realize new integrated devices for chemical and biosensing. ${ }^{3,4}$ Moreover, the LSP of small metallic particles can be coupled to a metallic film in order to perform complex operations on propagating SPP waves. For example, the utilization of periodically patterned metallic surfaces has already been demonstrated for SPP guiding and manipulation at the nanoscale. ${ }^{5-8}$

As all these devices are strongly sensitive to the light frequency, it could be interesting to dispose tunable particles to modify their frequency range. In this Letter we present the tunability properties of a single composite particle, as illustrated in Fig. 1. This object can be easily realized experimentally by classical lithography technics. It consists of a two-layer rectangular particle. The upper layer is made of gold, which supports the LSP mode; the lower layer is made of dielectric material. The resonance properties of this composite particle, when coupled to a metallic film, are investigated numerically, and it is shown that varying the dielectric spacer characteristics allows one to easily change the resonance wavelength. Calculations were performed by the Green's tensor method. ${ }^{9,10}$ Throughout the paper we consider a 50 $\mathrm{nm}$ thick gold film deposited on a silica substrate with permittivity $\epsilon=2.25$. The gold permittivity data are from Johnson and Christy. ${ }^{11}$ The structure is illuminated from underneath at normal incidence (elec- tric field propagating along the $z$ direction and polarized along the $x$ direction).

Let us consider first what happens when a rectangular metallic particle of thickness $t$ with a square basis of side $a$ is brought near the metallic slab, without a dielectric spacer. Figure 2 shows the evolution of the extinction spectrum for $a=100 \mathrm{~nm}$ and $t$ $=20 \mathrm{~nm}$ as a function of the distance $d$. In vacuum it is well known that such a particle possesses several LSP modes, whose number and positions depend on the particle aspect ratio, volume, and surrounding medium permittivity. ${ }^{12}$ For the present geometry, only one resonance appears in the range of the wavelength computed, as shown in Fig. 2. This mode is excited by an incident electric field polarized parallel to the long side $a$. Two distinct regimes can be observed when the separation is varied. When $d$ is larger than $80 \mathrm{~nm}$, no significant resonance shift occurs, and the particle can be considered to be placed in a homogeneous space. But for distances shorter than $80 \mathrm{~nm}$, a large shift toward IR occurs. In the limit of the contact between the particle and the film, no resonance occurs in the studied range. This tuning property is very interesting from an experimental point of view.

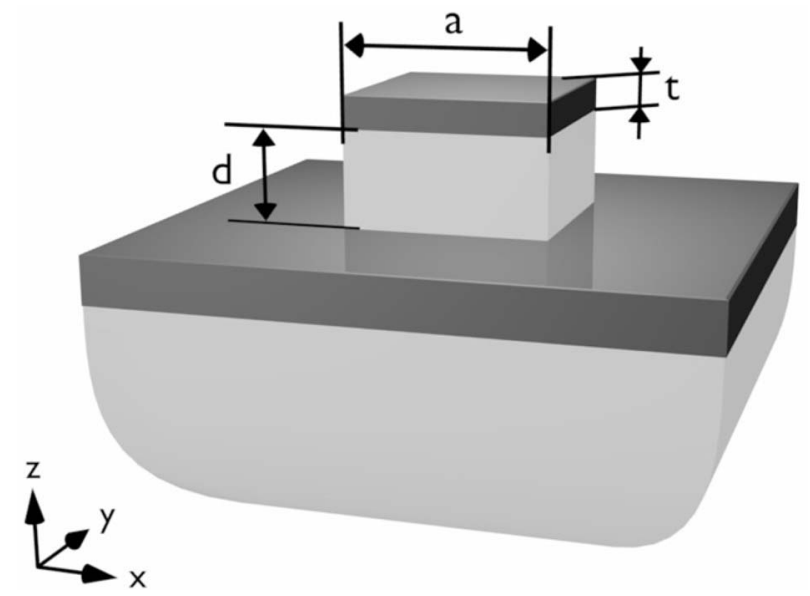

Fig. 1. Square-basis composite particle made of a metallic part of thickness $t$, a dielectric spacer of thickness $d$, and permittivity $\epsilon_{d}$. It is coupled to a 50 -nm-thick metallic slab deposited on a silica substrate. 


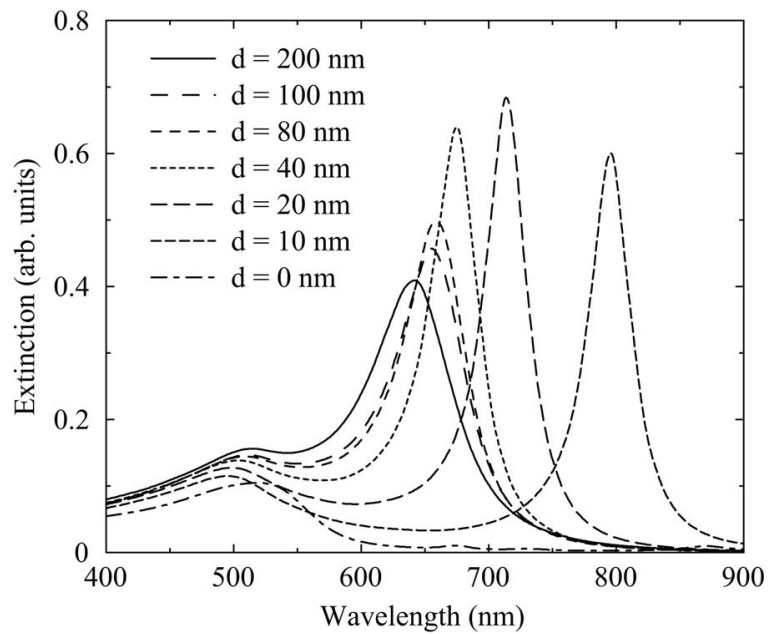

Fig. 2. Extinction spectrum for a particle with $t=20 \mathrm{~nm}$ and $a=100 \mathrm{~nm}$ in vacuum as a function of the distance $d$ from the metallic slab.

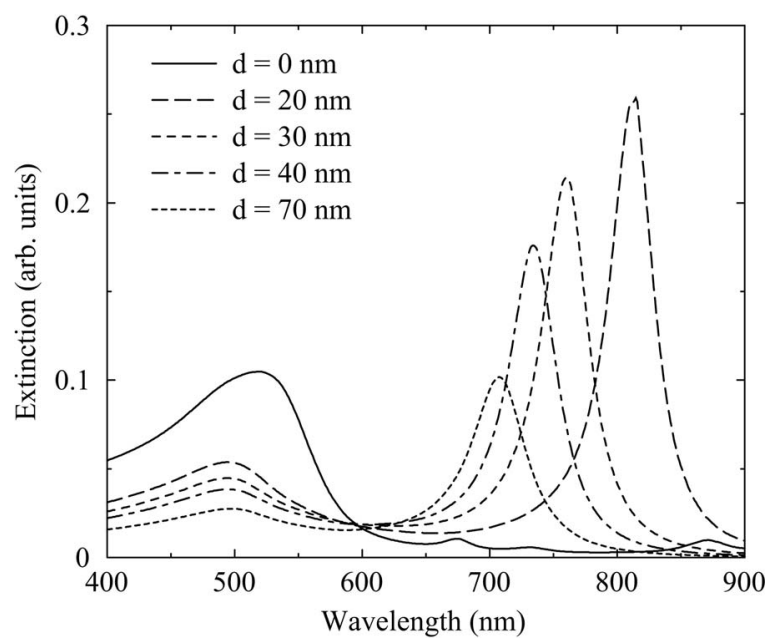

Fig. 3. Extinction spectrum for a metallic particle with $t$ $=20 \mathrm{~nm}$ and $a=100 \mathrm{~nm}$ for increasing values of the dielectric spacer thickness $d$ of permittivity $\epsilon_{d}=2.25$.

Indeed, it allows one to choose precisely the wavelength of the LSP mode of a metallic nanoparticle to tune it, for example, to a laser frequency or a molecular transition.

For practical realization the particle must be supported by a dielectric spacer, as shown in Fig. 1. The dielectric spacer permittivity $\epsilon_{d}$ becomes a new parameter to characterize the system. In the following, the metallic particle side will be set to $a=100 \mathrm{~nm}$, and its thickness to $t=20 \mathrm{~nm}$. We will focus on the parameters $d$ and $\epsilon_{d}$. The metallic film supports two SPP modes confined along each of its two interfaces. As we are interested in the LSP mode associated with the particle, the slab is illuminated from the glass by a plane wave in normal incidence. Hence no SPP wave can be directly excited by the illuminating field. The permittivity of the spacer is fixed to $\epsilon_{d}=2.25$. Figure 3 shows the evolution of the extinction spectrum when the spacer thickness $d$ is changed. If $d$ $=0 \mathrm{~nm}$, the resonance is shifted beyond $1000 \mathrm{~nm}$. However, a spacer thickness of some tens of nanometers is enough to bring it back below $900 \mathrm{~nm}$. For ex- ample, the resonance wavelength is located around $760 \mathrm{~nm}$ for $d=30 \mathrm{~nm}$. When the thickness increases from $20 \mathrm{~nm}$ to $70 \mathrm{~nm}$, the resonance wavelength shifts by more than $100 \mathrm{~nm}$. These results are qualitatively consistent with the experiment done with silver by Holland and Hall. ${ }^{13}$ Hence the plasmon resonance of the composite particle can be tuned by simply changing the thickness of the dielectric spacer $d$. This is important, as deposition of dielectric materials such as $\mathrm{SiO}_{2}$ or $\mathrm{TiO}_{2}$ can be controlled within a few nanometers.

It is interesting to compute the electric field in the polarization plane of the incidence wave, as presented in Fig. 4. The thickness of the dielectric spacer is $d=20 \mathrm{~nm}$. The gray scale corresponds to the amplitude of the electric field [Fig. 4(a)], whereas the arrows show the real and imaginary parts of the polarization vector [Fig. 4(b)]. The incident field is a plane wave at normal incidence, and its wavelength is $810 \mathrm{~nm}$, corresponding to the plasmon mode for this spacer thickness (Fig. 3). The incident electric field is polarized along the $x$ direction. Figure 4(a) shows that the electromagnetic energy is concentrated

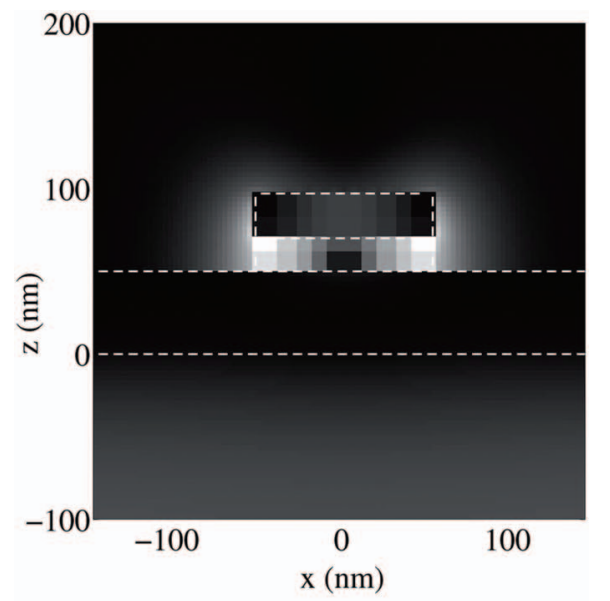

(a)

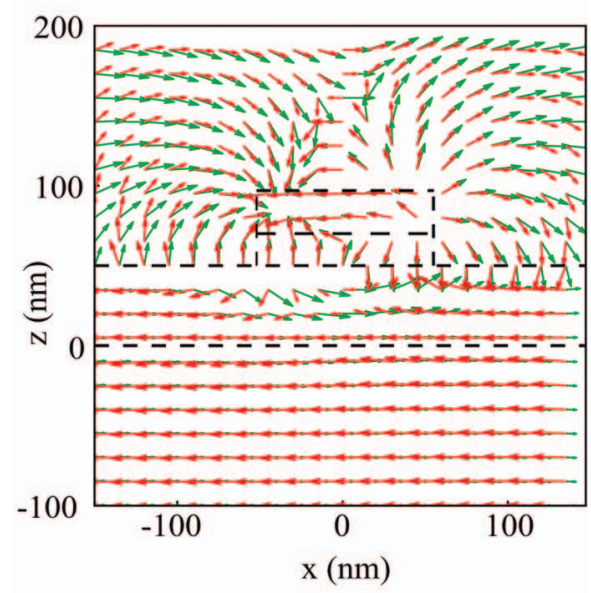

(b)

Fig. 4. Distribution of the (a) electric field amplitude and (b) polarization at $\lambda=810 \mathrm{~nm}$, the plasmon resonance wavelength for a 20-nm-thick spacer. The green (red) arrows represent the real (imaginary) part of the polarization vector. 
mainly inside the dielectric spacer. Moreover, the arrows in Fig. 4(b) show that the polarization of the electric field is strongly modified in the vicinity of the particle. The polarization is elliptical just above the metal-air interface, which is an indication that the light scattered by the resonant composite particle is coupled back to the SPP mode on the gold-vacuum interface of the film (the polarization of the SPP is elliptical at this interface). Additionally, the orientation of the arrows around the particle shows that the field scattered from the metallic part is of a dipolar nature, the dipole being oriented parallel to the interface. Moreover, this dipole is coupled to its image in the metallic medium.

The other experimental parameter that can be varied is the spacer permittivity. Figure 5 shows the evolution of the plasmon resonance wavelength as a function of the permittivity for three different values of the spacer thickness $d$. The permittivity ranges from 1.0 , the value of the vacuum, to 4.5 , which is slightly higher than the permittivity of $\mathrm{TiO}_{2}$. It appears that the resonance wavelength is quite sensitive to $\epsilon_{d}$, and that the sensitivity decreases when $d$ increases. These curves show that the dependency is almost perfectly linear, at least within the investigated parameter range. This behavior has its origin in the real part of the gold permittivity used, which is very linear in the $600-900 \mathrm{~nm}$ wavelength range. For $d=30 \mathrm{~nm}$, the tuning sensitivity is $\sim 60 \mathrm{~nm}$ per unit of $\epsilon_{d}$. This large effect might appear surprising, since only a small part of the environment of the metallic part is modified by the spacer. Actually, it is a consequence of the fact that most of the electromagnetic field is confined inside the spacer, as illustrated in Fig. 4.

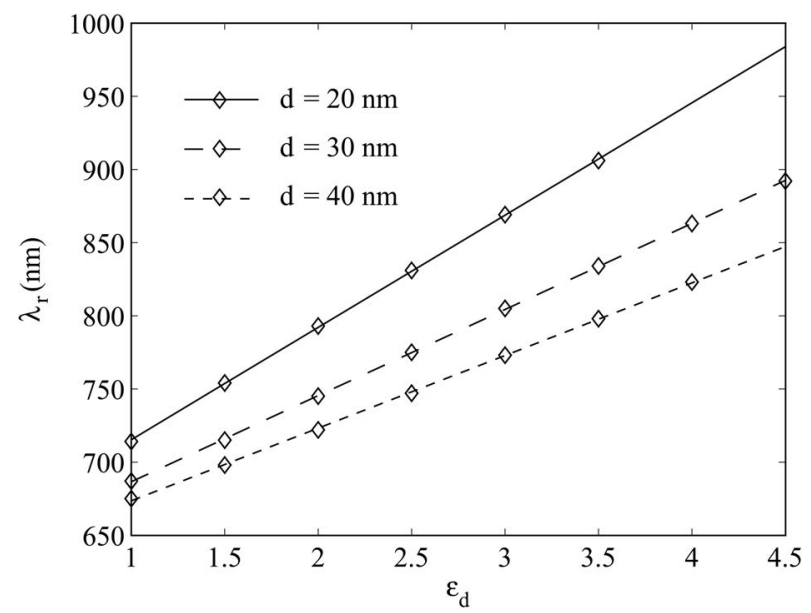

Fig. 5. Plasmon resonance wavelength as a function of the spacer permittivity $\epsilon_{d}$ for three different spacer thicknesses, $d$. Lines are linear fits of the data: $\lambda_{r}=77 \epsilon_{d}+639$ for $d=20 \mathrm{~nm}, \lambda_{r}=59 \epsilon_{d}+628$ for $d=30 \mathrm{~nm}$, and $\lambda_{r}=49 \epsilon_{d}+624$ for $d=40 \mathrm{~nm}$.
In conclusion, we have shown that the addition of a small dielectric spacer of a few tens of nanometers between a metallic particle and a metallic slab allows us to bring the LSP resonance back to the $700-900 \mathrm{~nm}$ window. Moreover, two easily controllable experimental parameters can be used to tune the resonance wavelength. Changing the spacer thickness by $50 \mathrm{~nm}$ shifts the resonance over more than $100 \mathrm{~nm}$, whereas increasing the value of the permittivity from 1.5 to 4.5 increases the resonance wavelength by $100 \mathrm{~nm}$ up to $200 \mathrm{~nm}$ depending on the spacer thickness. These two independent degrees of freedom are essential in an experimental realization in which all the parameters cannot be easily varied. Moreover, since all the plasmonic devices are wavelength sensitive, the spectral tunability of the composite particles presented in this Letter should make possible the realization of plasmonic systems operating at any given wavelength.

It is a pleasure to acknowledge extremely fruitful discussions with A.-L. Baudrion and J.-C. Weeber from Burgondy University and funding from the Information Science and Technology Network of Excellence Plasmonanodevices (FP6-2002-IST-1507879). G. Leveque's e-mail address is gaetan.leveque@epfl.ch.

\section{References}

1. M. G. Cottam and D. R. Tilley, Introduction to Surface and Superlattice Excitations (Cambridge U. Press, 1989).

2. C. F. Bohren and D. R. Huffman, Absorption and Scattering of Light by Small Particles (Wiley, 1983).

3. J. P. Kottmann, O. J. F. Martin, D. R. Smith, and S. Schultz, J. Microsc. 202, 60 (2001).

4. P. Ghenuche, R. Quidant, and G. Badenes, Opt. Lett. 30, 1882 (2005).

5. S. I. Bozhevolnyi, J. Erland, K. Leosson, P. M. W. Skovgaard, and J. M. Hvam, Phys. Rev. Lett. 86, 3008 (2001).

6. H. Ditlbacher, J. R. Krenn, G. Schider, A. Leitner, and F. R. Aussenegg, Appl. Phys. Lett. 81, 1762 (2002).

7. J.-C. Weeber, Y. Lacroute, A. Dereux, E. Devaux, T. Ebbesen, C. Girard, M. U. González, and A.-L. Baudrion, Phys. Rev. B 70, 235406 (2004).

8. J.-C. Weeber, M. U. González, A.-L. Baudrion, and A. Dereux, Appl. Phys. Lett. 87, 221101 (2005).

9. M. Paulus and O. J. F. Martin, J. Opt. Soc. Am. A 18, 854 (2001).

10. J. P. Kottmann and O. J. F. Martin, IEEE Trans. Antennas Propag. 48, 1719 (2000).

11. P. B. Johnson and R. W. Christy, Phys. Rev. B 6, 4370 (1972).

12. J. P. Kottmann, O. J. F. Martin, D. R. Smith, and S. Schultz, Phys. Rev. B 64, 235402 (2001).

13. W. R. Holland and D. G. Hall, Phys. Rev. Lett. 52, 1041 (1984). 